\title{
Evaluation De La Prematurite Superieure Ou Egale A 32 Semaine D'amenorhée A L'hopital Regional De Ziguinchor Au Sud Du Senegal (Afrique De L'ouest)
}

\author{
Diouf Francois Niokhor \\ Diallo Fatima Bintou
}

Service de pédiatrie à l'hôpital regional de Ziguinchor

Thiam Lamine

Kane Yaya

Service de pédiatrie à l'hôpital de la Paix de Ziguinchor

Ba Idrissa Demba

Ba Abouba

Faye Pape Mactar

Ndiaye Ousmane

Service de Pédiatrie de l'hôpital d'enfant Albert Royer Dakar/ Sénégal

Gueye Modou

Service de Pédiatrie de l'hôpital Abass DAO/ Sénégal

Diagne Ndeye Ramatoulaye

Service de Pédiatrie de l'hôpital d'enfant de Diamniadio

Doi: 10.19044/esj.2017.v13n36p325 URL:http://dx.doi.org/10.19044/esj.2017.v13n36p325

\begin{abstract}
Introduction: Prematurity is one of the leading causes of neonatal death in Africa. The objective of this work was to assess the preterm birth at the pediatric service of the regional hospital of Ziguinchor. Material and methods: This was a prospective, descriptive and analytical study of the case of hospitalized newborns whose age was between 32 SA to 36SA +6 days. The study was conducted from May 21, 2013 to May 21, 2014. We included all premature infants at age $\geq 32$ SA.Those presenting a malformation were not included. We studied maternal socio-demographic, obstetric and neonatal parameters. Results: We have identified 140 newborns out of a total of 342 Preterm births and 2292 maternity births, a prevalence of $40.9 \%$ compared to preterm infants and $6.10 \%$ in relation to all births. 51.4 percent of mothers came from urban areas, 63.5 percent were between 20 and 34 years of age, not attending school in 45.7 percent, married in 72.9 percent and without work in 94.3 percent. The average gestures represented 3.09 . Arterial
\end{abstract}


hypertension was the most common medical condition $(67.7 \%)$. The followup was done by a midwife $(82.1 \%)$ and $23.5 \%$ had received at least 4 NPC. Eclampsia and pre-eclampsia (48.9\%) and RPM (42.8\%) were common obstetric pathologies. The delivery was by caesarian (51.4\%), hospital (92.1\%), cephalic presentation (80\%), with an average weight of $1816.79 \mathrm{~g}$. An RCIU (16.4\%), an RPM (61.9\%). At birth, a DR (16.4\%) was noted due to $\mathrm{MMH}(43.7 \%)$ and transitory tachypnea (50\%). Asphyxia was reported in $3.5 \%$. During hospitalization (6.7 days on average), $6.4 \%$ had RD caused by infection (77.7\%). Other complications were infection (64.4\%), hypoglycemia $(28.5 \%)$, digestive hemorrhage $(7.01 \%)$. The fatality rate was $7.9 \%$ due to infection (63.3\%), DR (18\%), haemorrhage (9\%). The anthropometric measurements at the exit: P $1887.9 \mathrm{~g}, \mathrm{~T}: 38.3 \mathrm{~cm}, \mathrm{PC}: 30.9 \mathrm{~cm}$; at 1 month P: $2387.1 \mathrm{~g}, \mathrm{~T}: 46.6 \mathrm{~cm}, \mathrm{PC}: 32.8 \mathrm{~cm}$; at 30 months P: $12.1 \mathrm{~kg}, \mathrm{~T}$ : $89.5 \mathrm{~cm}$, PC: $48.4 \mathrm{~cm}$. Conclusion: Moderate preterm birth accounts for almost half of the cases of prematurity in our series. Their optimal management would go through a better obstetric-neonatal collaboration but above all by the installation of kangaroo mother units

Keywords: Prematurity, moderate, epidemiology, prognosis

\section{Résumé}

Introduction : La prématurité est l'une des principales causes de décès néonataux en Afrique. L'objectif était de faire le bilan de la prématurité $\geq$ à $32 \mathrm{SA}$ dans le service de pédiatrie de l'hôpital régional de Ziguinchor. Matériel et Méthodes: Il s'agissait d'une étude prospective, descriptive et analytique de dossier de nouveau-nés hospitalisés dont l'âge était compris entre $32 \mathrm{SA}$ à 36SA + 6jours. L'étude s'est déroulée du 21 Mai 2013 au 21 Mai 2014. Nous avons inclus tous les nouveau-nés prématurés dont l'âge était $\geq$ à $32 \mathrm{SA}$, n'ont pas été inclus ceux qui présentaient une malformation. Nous avons étudié les paramètres sociodémographiques maternels, obstétricaux et néonataux. Résultats : Nous avons répertorié 140 nouveau-nés sur un total de 342 accouchements prématurés et 2292 naissances à la maternité soit une prévalence de 40,9\% par rapport aux prématurés et $6,10 \%$ par rapport à l'ensemble des naissances. $51,4 \%$ des mères provenaient d'une zone urbaine, 63,5\% avaient un âge compris entre 20 et 34 ans, non scolarisées dans 45,7\%, mariées dans 72,9\% et sans activité professionnelle dans 94,3\%. La gestité moyenne était de 3,09. L'hypertension artérielle était la pathologie médicale la plus fréquente $(67,7 \%)$. Le suivi était fait par une sage femme $(82,1 \%)$ et $23,5 \%$ avaient fait au moins $4 \mathrm{CPN}$. L'éclampsie et la pré éclampsie (48,9\%) et la RPM (42,8\%) étaient les pathologies obstétricales courantes. L'accouchement était fait par voie haute $(51,4 \%)$, à l'hôpital $(92,1 \%)$, en présentation céphalique 
(80\%) avec un poids moyen de 1816,79 g. Un RCIU (16,4\%), une RPM $(61,9 \%)$. A la naissance, une DR $(16,4 \%)$ a été notée causée par la $\mathrm{MMH}$ $(43,7 \%)$ et la tachypnée transitoire $(50 \%)$. Une asphyxie était notée dans 3,5\%. En cours d'hospitalisation (6,7 jours en moyenne), 6,4\% présentaient une DR causée par l'infection $(77,7 \%)$. Les autres complications étaient l'infection (64,4\%), l'hypoglycémie (28,5\%), l'hémorragie digestive $(7,01 \%)$. Le taux de létalité était de 7,9\% causée par l'infection $(63,3 \%)$, la DR (18\%), l'hémorragie (9\%). Les mesures anthropométriques moyennes à la sortie $\mathrm{P}: 1887,9 \mathrm{~g}, \mathrm{~T}: 38,3 \mathrm{~cm}, \mathrm{PC}: 30,9 \mathrm{~cm}$; à 1 mois $\mathrm{P}: 2387,1 \mathrm{~g}, \mathrm{~T}$ : $46,6 \mathrm{~cm}, \mathrm{PC}: 32,8 \mathrm{~cm}$; à 30 mois $\mathrm{P}: 12,1 \mathrm{~kg}, \mathrm{~T}: 89,5 \mathrm{~cm}, \mathrm{PC}: 48,4 \mathrm{~cm}$. Conclusion : La prématurité modérée occupe quasiment la moitié des cas de prématurés dans notre série. Leur prise en charge optimale passerait par une meilleure collaboration obstétrico-néonatale mais surtout par l'installation d'unités mères kangourou

Mots clés: Prématurité, modérée, épidémiologie, pronostic

\section{Introduction}

Le faible poids en général, la prématurité en particulier, est l'une des principales causes de décès néonataux en Afrique. Elle vient en troisième position derrière l'asphyxie et les infections (Cissé, 1998 Pattinson, 2004 Amri, 2008 Bobossi, 2000). La prise en charge de la prématurité est très difficile dans nos pays en voie de développement compte tenu de la faiblesse du plateau technique, la non disponibilité de médicaments essentiels et l'insuffisance de formation du personnel médical et paramédical. L'un des objectifs majeurs du millénaire (OMD n4 pour le développement) était la réduction de la mortalité des enfants de moins de 5 ans. En effet la mortalité néonatale occupe plus de $70 \%$ de la mortalité infantino-juvénile dans la plupart des pays sub-sahariens (Cissé, 1998 Pattinson, 2004 Amri, 2008 Bobossi, 2000). Force est de croire que cet objectif, malgré les progrès réalisés dans beaucoup de pays en voie de développement, n'a pas été atteint. L'identification des facteurs associés à la naissance de ces populations à risque dans nos structures de santé pourrait optimisée cette réduction.

L'objectif de ce travail était de faire le bilan de la prématurité supérieure ou égale à $32 \mathrm{SA}$ dans le service de pédiatrie de l'hôpital régional de Ziguinchor.

\section{Patients et methodes}

\section{Cadre et type d'étude}

Il s'agissait d'une étude prospective, descriptive et analytique de dossier de nouveau-nés prématurés hospitalisés dont l'âge était compris entre 32 SA et 36SA + 6jours. L'étude s'est déroulée du 21 Mai 2013 au 21 
Mai 2014 soit une durée de 12 mois. Elle a eu lieu dans le service de la pédiatrie de l'hôpital régional de Ziguinchor qui accueille les enfants de 0 à 15 ans. C'est un service qui est constitué de 4 compartiments : une unité de néonatologie ( 0 à 1 mois) avec une capacité de 21 lits (13 lits ou berceaux d'hospitalisation, 4 couveuses, 4 tables chauffantes de réanimation) ; une salle d'urgence pour les nourrissons et les grands enfants ( 2 mois à 15 ans) capacité 10 lits; une salle de convalescence ou salle d'allaitement avec 10 lits ; trois cabines individuelles.

\section{Patients}

\section{Critères d'inclusion}

Nous avons inclus tous les nouveau-nés prématurés « in born et out born » hospitalisés dans l'unité de néonatologie dont l'âge gestationnel était compris entre $32 \mathrm{SA}$ et $36 \mathrm{SA}+6$ jours.

\section{Critères de non inclusion}

Nous n'avons pas inclus les prématurés qui ne répondaient pas aux critères sus -cités et ceux qui présentaient une malformation.

\section{Collecte des données}

Les données ont été recueillies à partir des dossiers d'hospitalisation. Elles ont été consignées sur une fiche de collecte de données pré - établie. Nous avons analysé les paramètres suivants :

- données sociodémographiques maternelles (âge, niveau d'instruction, statut matrimonial, Activité professionnelle, origine géographique)

- antécédents gynécologiques et/ou obstétricaux, médicaux et chirurgicaux

- données sur la grossesse (nombre de CPN, suivi de la grossesse, pathologies obstétricales, type de transfert)

- données sur l'accouchement et données néonatales (âge gestationnel, voie d'accouchement, lieu d'accouchement, présentation, score d'APGAR et notion de réanimation, poids, sexe, retard de croisance inta utérin (RCIU), rupture prématurée des membranes (RPM), aspect liquide amniotique, )

- données sur l'hospitalisation et la prise en charge: durée d'hospitalisation, mode d'hospitalisation, complications néonatales, mode d'alimentation, létalité, suivi à distance à 1 mois et 30 mois

\section{Définition des paramètres}

Le terme de la grossesse était déterminé à partir de la date des dernières règles, ou la mesure de la hauteur utérine, ou encore lors de l'échographie précoce. A défaut, la grossesse était datée après la naissance en se basant sur le score neuro-morphologique de BALLARD (Ballard, 
1979). L'âge des mères était déterminé à l'accouchement. Elles étaient réparties en trois groupes : celles dont l'âge était inférieur à 20 ans, celle dont l'âge était entre 20 et 34 ans et le troisième groupe dont l'âge était supérieur ou égale à 35 ans. L'origine géographique était subdivisée en zone urbaine (département de Ziguinchor), suburbaine (autres départements), rurale (village). Les lieux d'accouchement répertoriés étaient: hôpital, domicile, poste de santé, clinique privée. Le mode d'hospitalisation consistait à préciser si le nouveau-né était hospitalisé en incubateur fermé ou ouvert. Le niveau d'instruction des mères était reparti en quatre groupes (jamais scolarisée, niveau primaire, niveau secondaire et niveau supérieur). La parité était repartie en 4 groupes : les primipares ayant un enfant, les pauci pares celles dont la parité était comprise entre 2 et 3 pares, les multipares entre 4 et 5 pares et enfin les grande multipares dont la parité était supérieure ou égale à 6.Les constantes anthropométriques du prématuré ont été comparées aux courbes de croissance de Leroy et évaluées dans l'heure qui suivait la naissance. La souffrance fœetale aigue était définie par un sore d'APGAR inférieur à 7 à $5 \mathrm{mn}$ de vie. Etant données les difficultés rencontrées quand au suivi mensuel de nos prématurés liés «aux perdus de vue» (changement de répertoire téléphonique, origine transfrontalière, moyens de déplacement), les modalités du suivi à distance consistaient à prendre les mesures anthropométriques (Poids, Taille, Périmètre crânien) à 1 mois et 30 mois ainsi que la recherche de troubles du langage et de séquelles neurologiques à 30 mois. Pour ce faire, nous avons rappelé à ces dates par téléphone, toutes les mères qui étaient disponibles pour venir au rendez-vous. Nous avons défini comme trouble du langage tout prématuré qui présentait des troubles de l'élocution. Les séquelles neurologiques correspondaient aux troubles moteurs et/ou sensoriels.

\section{Analyse des données}

Les données étaient analysées par le logiciel EPI INFO version 7.

\section{Resultats}

Durant la période de l'étude, nous avons enregistré 2292 accouchements. Parmi ces nouveau-nés nous avons répertorié 342 accouchements prématurés soit une prévalence de la prématurité de $15 \%$. Les accouchements prématurés dont l'âge gestationnel était supérieur ou égal à 32 SA constituaient 140 cas, ce qui fait une prévalence par rapport aux prématurés de $41 \%$ et par rapport à l'ensemble des naissances de $6 \%$.

\section{Données socio-démographiques}

Nos parturientes avaient un âge compris entre 20 et 34 ans dans $63,5 \%$ des cas. Elles provenaient d'une zone urbaine dans plus de la moitie 
des cas $(51,4 \%)$. La quasi-totalité des parturientes $(94,3 \%)$ n'avait pas une activité professionnelle et $45,7 \%$ étaient analphabètes. Près de $3 / 4$ des mères (73\%) étaient mariées. La gestité moyenne était de 3,04 avec des extrêmes (1 - 9), la parité moyenne de 3,16 avec des extrêmes (1 - 9). Chez nos prématurés nous avons noté une prédominance masculine avec un sexe ratio de 1,09 .

\section{Données sur les antécédents maternels}

L'hypertension artérielle, le paludisme, l'accouchement prématuré et l'avortement étaient les pathologies maternelles les plus fréquemment rencontrées avec respectivement $67,7 \%, 16,1 \%, 55,1 \%$ et $24,48 \%$. Les pathologies obstétricales les plus retrouvées étaient les anomalies vasculaires et la RPM avec respectivement $49 \%$ et $42,8 \%$.

\section{Données sur la grossesse et données péri-nataux}

Tableau 1 : Données sur la grossesse

\begin{tabular}{|c|c|c|}
\hline & Nombre de cas (N=140) & Pourcentage(\%) \\
\hline Nombre de CPN & 33 & 23.57 \\
$\geq 4$ & 103 & 73.57 \\
1 à 3 & 4 & 2.85 \\
0 & 115 & 82.10 \\
Suivi de la grossesse & 5 & 3.6 \\
Sage femme & 12 & 8.6 \\
Obstétricien & 8 & 5.7 \\
Infirmier & 54 & 38.6 \\
Autres & 53 & 37.9 \\
Détermination de l'AG & 7 & 5 \\
DDR & 26 & 18.6 \\
Echographie précoce & & \\
HU & 128 & 91.4 \\
Score de maturation & 6 & 4.3 \\
Transfert des mères & 6 & 4.3 \\
A pieds & & \\
Ambulance & 68 & 48.6 \\
Taxi & 66 & 47.12 \\
\hline Nombre de foetus & 3 & 2.14 \\
Monofoetal & 3 & 2.14 \\
Gémellaire & &
\end{tabular}

Tableau 2 : données péri-nataux

\begin{tabular}{|c|c|c|}
\hline & Nombre de cas N=140 & Pourcentage(\%) \\
\hline Voie d'accouchement & 72 & 51.4 \\
Haute & 68 & 48.6 \\
Basse & & \\
\hline
\end{tabular}




\begin{tabular}{|c|c|c|}
\hline Présentation & 112 & 80 \\
Céphalique & 24 & 17 \\
Siège & 4 & 3 \\
Face & & \\
Lieu d'accouchement & 4 & 2.9 \\
Domicile & 129 & 92.1 \\
Hôpital & 6 & 4.3 \\
Poste de santé & 1 & 0.7 \\
Clinique privée & & \\
Score d'Apgar à M1 & 95 & 67.85 \\
$\geq 7$ & 40 & 28.57 \\
4à6 & 5 & 3.57 \\
\hline 3 (Asphyxie) & 45 & 32.15 \\
Oui & 95 & 67.85 \\
non & & \\
Notion de réanimation & 73 & 52.14 \\
F & 67 & 47.86 \\
\hline Sexe (R=1.09) & 67 & 47.9 \\
F 35 & 73 & 52.1 \\
\hline Age gestationnelle(SA) & &
\end{tabular}

Une détresse respiratoire et neurologique à la naissance était présente respectivement dans $16,42 \%$ et $5,71 \%$ des cas. Les principales causes de la détresse respiratoire à la naissance étaient la maladie des membranes hyalines $(\mathrm{MMH})(43,75 \%)$ et la tachypnée transitoire (50\%).

Les poids de naissance variaient entre $800 \mathrm{~g}$ et $2600 \mathrm{~g}$. La tranche de poids de $1500 \mathrm{~g}$ à $2000 \mathrm{~g}$ était la plus représentée $(52,85 \%)$, avec un poids moyen de $1816,7 \mathrm{~g}$.

Chez nos prématurés nous avons noté une prédominance masculine avec un sexe ratio de 1,09 .

\section{Données sur l'hospitalisation et la prise en charge}

Le nombre de jours d'hospitalisation variait entre un et seize jours avec une moyenne de 6,72 jours. Les nouveau-nés admis étaient hospitalisés sur incubateur ouvert ou table chauffante dans 19,30\%, sur incubateur fermé ou couveuse dans $1,40 \%$. Une détresse respiratoire en cours d'hospitalisation était présente dans $6,43 \%$ des cas et la principale cause était l'infection (77,7\%). Les autres complications retrouvées étaient l'hémorragie 7,01\%, l'hypoglycémie $28,57 \%$ et l'infection $64,42 \%$. Le taux de mortalité était de $7,90 \%$. Les principales circonstances de décès étaient l'infection, la détresse respiratoire, et l'hémorragie avec respectivement $63,60 \%, 18,20 \%$ et $9,10 \%$. Les nouveau-nés recevaient un allaitement artificiel à la naissance dans plus de la moitie des cas $(52,2 \%)$. Le poids moyen à la sortie était de $1887,9 \mathrm{~g}$ 
(extrêmes: 1200 - 2800) avec une taille moyenne de 38,34 cm (extrêmes : 30 - 52) et un périmètre crânien (PC) moyen de 30,98 cm (extrêmes : 27 - 37).

\section{Données selon le suivi}

Pour les prématurés qui ont pu être suivis à un mois de vie $(30 \%$ des cas) le poids moyen était de 2387,14 g (extrêmes : 1300 - 1800), la taille moyenne de $46,65 \mathrm{~cm}(40-55)$ et le PC moyen de $32,81 \mathrm{~cm}(28-37)$. Le suivi à 30 mois a été évalué pour $26,42 \%$ des cas avec un poids moyen de $12,16 \mathrm{~kg}(7,5$ - 14,1), taille moyenne de $89,58 \mathrm{~cm}(83-99)$ et PC moyen de $48,49 \mathrm{~cm}(45,5-51,5)$. Le langage a été évalué à 30 mois dans notre cohorte dans $26,42 \%$ des cas. Parmi eux $8,11 \%$ présentaient un trouble du langage. Parmi nos prématurés suivis à 30 mois, seuls $2,70 \%$ présentaient des séquelles neurologiques.

\section{Discussion \\ Prévalence}

Dans notre travail, la prévalence de la prématurité dont l'âge gestationnel était supérieur ou égal à $32 \mathrm{SA}$ était de $41 \%$ par rapport à l'ensemble des prématurés. Ce pourcentage est faible comparé à celui retrouvé dans le monde et en Afrique avec respectivement $80 \%$ et $75 \%$ (Born too soon, 2012). Par ailleurs Ly Cheong et al. en Australie avaient trouvé $84 \%$ de prématurité modérée (Jeanie, 2012), Gueye et al. au Sénégal 52\%(Gueye, 2011), Valery et al. en France 75\% (Valery, 2015). Katz et al. en Asie, Afrique Sub-saharienne et Amérique latine avaient retrouvé un pourcentage qui variait entre 50 et 96\% (Katz, 2013 et Ye D et al. au Burkina Faso $68 \%$ (YE D, 1999). Cette faible prévalence que nous avons retrouvé dans notre étude comparé au reste du monde pourrait s'expliquer par le problème de suivi des grossesses dans la localité lié à l'enclavement et au déficit en personnel qualifie.

La prévalence de la prématurité supérieur ou égale à $32 \mathrm{SA}$ était de $6,10 \%$ sur l'ensemble des naissances. Une étude faite en France sur des accouchements prématurés dont l'âge gestationnel était supérieur à 34 SA montre une prévalence de 4,9\% (Jeanie, 2012).

Toutes ces prévalences sont celles des unités de néonatologie, qui ne reflètent pas vraiment la prévalence de la prématurité dans la population générale.

\section{Données socio-démographiques}

Dans notre étude, la moitié $(51,4 \%)$ des parturientes provenait de la ville de Ziguinchor (zone urbaine) ceci s'explique par le fait que l'accès à l'hôpital était difficile pour les femmes rurales (manque de moyens, village enclavé, routes défectueuses). A l'opposé, dans l'étude de Ndiaye et al. les 
parturientes provenaient plus du milieu rural (Ndiaye, 2006). Une étude faite à Dakar par Gueye et al. avait trouvé que $80 \%$ des femmes provenaient de la banlieue (Gueye, 2011). La relation entre niveau socioéconomique et risque d'accouchement prématuré est assez étroite. Ainsi nous avons retrouvé dans notre travail que la quasi-totalité $(94,3 \%)$ des parturientes n'avait pas d'activité professionnelle. Ce même constat a été fait à l'hôpital Abass Ndao par Gueye et al. qui avaient retrouvé 90,5\% des mères avec un niveau socioéconomique bas (Gueye, 2011). D’autres études ont confirmé cette relation entre accouchement prématuré et niveau socio-économique des mères (Diallo, 1998 Lejeune, 2008). L’âge des parturientes dans notre série était dans plus de la moitié des cas $(63,5 \%)$ compris entre 20 et 34 ans. Cette tranche d'âge est dans l'intervalle d'âge de procréation qui varie entre 15 et 49 ans selon l'EDS 2014. D'autres auteurs pour cette même tranche d'âge avaient trouvé des pourcentages moins élevés Ndiaye et al. 53\% (Ndiaye, 2006); Ouédraogo et al. 46,5\% (Ouédraogo, 2013); Gueye et al. 32,1\% (Gueye, 2011). Le niveau d'instruction des mères est corrélé au risque de naissance prématuré. Dans notre série $45,7 \%$ des mères étaient analphabètes et $34,3 \%$ avaient un niveau d'instruction faible. Ce même constat a été fait par Ouédraogo et al. (Ouédraogo, 2013). Au Togo, une étude retrouvait près de $40 \%$ des femmes avec un faible niveau d'instruction (Balaka, 2002). Nous avons constaté que la prématurité supérieure ou égale à 32SA dans notre travail était plus fréquente chez les parturientes dont la parité était comprise entre 1 et $3(63,6 \%)$. Ce même constat a été fait au Brésil $(83,7 \%)$, au Philippines (64,2\%), en Afrique du sud (84,5\%) et en Inde $(64,9 \%)$ (Caroline, 2015). Prazuck et al. retrouvaient une association plus étroite avec la primiparité (Prazuck, 1993). L'association entre multiparité et la prématurité est rapportée dans beaucoup de travaux (Cissé, 1998 Ezechi, 2003). Ndiaye et al. ont rapporté dans son étude que la multiparité est significativement associée à la prématurité (Ndiaye, 2006).

\section{Données sur les antécédents maternels}

Dans notre travail un ou plusieurs antécédents gynéco-obstétricaux étaient retrouvés chez $35 \%$ de nos mères dominés par 1'accouchement prématuré et les avortements avec respectivement 55,11\% et 24,48\%. Diouf et al. à Dakar avaient retrouvé une proportion similaire (31,9\%) d'antécédent gynéco-obstétricaux mais avec une prédominance des avortements (53\%) (Diouf, 2016). Balaka et al. au Togo avaient noté que 11,3\% de mères de prématuré avaient des antécédents d'avortement provoqué (Balaka, 2002). Diallo avait trouvé dans son travail que le risque d'accouchement prématuré était multiplié par 12 en cas d'antécédent de mort-né et par 7 en cas d'antécédent d'accouchement prématuré (Diallo, 1998). Des antécédents médicaux étaient retrouvés chez $22,14 \%$ de nos mères et étaient dominé par 
l'hypertension artérielle $(67,74 \%)$ et le paludisme $(16,12 \%)$. Le même constat a été fait par Diouf et al. qui avaientt retrouvé $23 \%$ d'antécédent médical dominé par l'hypertension artérielle (37,1\%) suivi de la drépanocytose (20\%) (Diouf, 2016). D'autres auteurs ont retrouvé l'hypertension artérielle comme antécédent médical dans seulement 9,7\% (Gueye, 2011) et 4,6\% (Ouédraogo, 2013). L'hypertension artérielle est largement reconnue comme étant un facteur de risque de survenue de la prématurité (YE D, 1999).

\section{Données sur la grossesse}

Dans notre série plus de $3 / 4$ des parturientes avait fait moins de 4 CPN contrairement aux recommandations du Sénégal. Selon Cissé et al. la fréquence des CPN est fortement corrélée à la prématurité, en effet dans son travail il avait démontré que $25 \%$ des femmes qui n'avaient jamais bénéficié de consultations prénatales avaient donné naissance à un prématuré (Cissé, 1998). Selon Prazuck et al. le nombre de CPN inférieur à 3 était le facteur le plus prédictif de prématurité (Prazuck, 1993). Ces résultats sont superposables à ceux rapportés dans la plupart des études au Sénégal et en Afrique sub-saharienne : 84\% pour Gueye et al. (Gueye, 2011), 93,1\% pour Ndiaye et al. (Ndiaye, 2006), 72,7\% pour Ouédraogo et al. (Ouédraogo, 2013 ) et $66,6 \%$ pour Balaka et al. (Balaka, 2002). Dans notre étude les grossesses multi fœetales représentaient près de la moitié des cas $(48,58 \%)$ d'autres auteurs ont trouvé des pourcentages moins élevés Amri et al. 31,8\% (Amri, 2008) et Gueye et al. 31,7\% (Gueye, 2011). Il existe une relation étroite entre accouchement prématuré et grossesse multi fotale. D'autres études ont trouvé des pourcentages assez représentatifs de la relation entre prématurité et grossesse multi fœtale: France 19,2\% (Lejeune, 2008), Ouagadougou 14,76\% (Ouédraogo, 2013).

Le suivi de la grossesse chez nos parturientes était fait dans la majeure partie des cas par une sage-femme $(82,10 \%)$. Ce même constat a été fait par certains auteurs (Gueye, 2011 Diouf, 2016 Ouédraogo, 2013).

Dans notre série, $35 \%$ des femmes présentaient des complications au cours de leur grossesse, dominées par les anomalies vasculaires $(48,97 \%)$ et de la rupture prématurée des membranes $(42,85 \%)$.

Ces complications expliquent une grande partie des naissances prématurées comme l'ont retrouvé d'autres auteurs mais dans des proportions moins importantes : Cissé 29,8\% (Cissé, 1998), Ouédraogo et al. $36,8 \%$ (Ouédraogo, 2013), Gueye et al. $20 \%$ et Valery et al. $28,9 \%$ et 25\% (Gueye, 2011 Valery, 2015). 


\section{Données péri-néonatales}

Un retard de croissance intra-utérin était associé à la prématurité dans $16,4 \%$ des cas dans notre série. Le taux de RCIU associée à la prématurité était élevé à $77 \%$ selon Ndiaye et al. (Ndiaye, 2006), 96,9\% pour Gueye et al. (Gueye, 2011), 46\% pour Amri et al. en Tunis (Amri, 2008), 33\% pour Katz et al. en Asie du sud (Katz, 2013) et 71\% pour Katz et al. en Amérique latine (Katz, 2013). L'association prématurité et RCIU est fréquente car les étiologies de ces 2 entités sont souvent intriquées. Le taux bas retrouvé dans notre étude pourrait s'expliquer par l'état nutritionnel (EN) de la maman qui est une des facteurs de risque de RCIU. En effet, l'EN des mères est meilleur dans cette zone du sud du Sénégal où la disponibilité alimentaire pose moins de problème que dans le reste du pays à cause de sa pluviométrie. L'âge de la grossesse dans notre travail était supérieur à $34 \mathrm{SA}$ dans $65,8 \%$ des cas. Des études faites en Australie montrent que $80 \%$ des prématurés modérés naissent après 34 SA (Jeanie, 2012). La césarienne était la voie de naissance pour $51,4 \%$ de notre population d'étude. Ce taux élevé dans notre cohorte pourrait s'expliquer par le fait que la maternité du CHRZ est la structure de référence ou se trouve le seul gynécologue de la région habilité à effectuer les césariennes. Par ailleurs les références tardives des parturientes par faute de moyens des structures périphériques fait que la plupart arrive à la maternité avec des complications nécessitant une césarienne en urgence. Cette proportion de naissance par voie césarienne était moins importante pour Gueye et al. 26,1\% (Gueye, 2011), Amri et al. 24,8\% (Amri, 2008), Ouédraogo et al. 21,8\% (Ouédraogo, 2013) et Diouf et al. 18,3\% (Diouf, 2016). La quasi-totalité de nos parturientes $(92,1 \%)$ avait accouché dans l'hôpital (« in Born »). Seuls 2,90\% des accouchements avaient eu lieu à domicile, taux qui est inférieur aux données de l'EDS 5 du Sénégal en 2010 (27\%) (EDS-MICS, 2012). Dans l'étude de Amri et al. en Tunisie, seuls 3\% étaient nés à domicile (Amri, 2008). Diouf et al. au Sénégal avaient trouvé 13,4\% (Diouf, 2016). La présentation de nos prématurés était céphalique dans $80 \%$ des cas. Ce pourcentage se rapproche des données de Diouf et al. 87,4\% (Diouf, 2016). Le rôle de la rupture des membranes dans le déclenchement spontané du travail est bien connu. Nous avons trouvé dans notre série la présence d'une rupture prématurée des membranes dans 15\% des cas. Ce taux est largement inférieur à ceux trouvés dans la littérature (Born too soon, 2012 Caroline, 2015 Gueye, 2011). Nous avons noté une prédominance masculine avec sexe ratio de 1,09, comme d'autres auteurs koko et al. au Gabon (Koko, 2002), Gueye et al. au Sénégal (Gueye, 2011) et Amri et al. en Tunisie (Amri, 2008). Cependant certains retrouvent une prédominance féminine : (Diouf, 2016 Ndiaye, 2006 Ouédraogo, 2013). Des difficultés d'adaptation à la naissance avec un score d'APGAR $<7$ étaient présentes dans $32,15 \%$ des cas. Ainsi $32,15 \%$ de nos nouveau-nés 
prématurés avaient été réanimés. Parmi ces nouveau-nés réanimés dans notre série 3,57\% présentaient une asphyxie. Ce faible pourcentage par rapport à certains auteurs (Amri, 2008 Cissé, 1998 Gueye, 2011 Diouf, 2016 Ouédraogo, 2013) s'expliquent par le fait que notre étude concernait la prématurité modérée qui pose moins de problème d'adaptation à la vie extra utérine.

Dans notre étude, une détresse respiratoire à la naissance était présente dans 16,42\% des cas, alors que ce taux était de 75\% chez Amri et al. (Amri, 2008), 89,7\% chez Gueye et al. et 49,6\% chez Diouf et al. (Diouf, 2016). Cette différence dans les proportions retrouvées s'explique par la population d'étude différente. En effet plus la prématurité est grande plus le nouveau-né présente des difficultés respiratoires. La cause principale de la détresse respiratoire à la naissance retrouvée chez nous était la tachypnée transitoire avec $50 \%$ suivie de la maladie des membranes hyalines avec 43,7\%. Ce même constat a été fait par d'autres auteurs (Gueye, 2011 Diouf, 2016). La tranche de poids $1500-2000$ g était la plus représentée $(87,85 \%)$ avec des extrêmes de $800 \mathrm{~g}$ et $2600 \mathrm{~g}$. Cette tranche comparée aux courbes de croissance, montre que la moitié de nos nouveau-nés avaient un poids normal.

\section{Données sur l'hospitalisation et la prise en charge}

Le nombre de jour d'hospitalisation variait dans notre travail entre un et seize jours avec une moyenne de 6,72 jours. La plupart de nos prématurés n'avait pas nécessité d'incubateur pour leur hospitalisation (79,3\%) contrairement à d'autres études (Gueye, 2011 Diouf, 2016). En cours d'hospitalisation $6,43 \%$ des cas présentaient une détresse respiratoire. La cause de la détresse respiratoire était dominée par l'infection avec 77,77\% des cas. Ce pourcentage était supérieur à ceux retrouvé par Gueye et al. et Diouf et al. qui étaient respectivement de 21,5\% et 68,2\% (Gueye, 2011 Diouf, 2016). Les autres complications répertoriées dans notre série étaient répartis comme suit: infection $(64,42 \%)$, hypoglycémies $(28,57 \%)$ et hémorragies digestives (7,01\%). Ces complications classiques de la prématurité avait été retrouvées par d'autres auteurs (Amri, 2008 Gueye, 2011 Diouf, 2016). Nous avons recensé un taux de mortalité de 7,90\%. Ce taux est faible comparé aux études réalisées à Abass Ndao (58,8\%) (Gueye, 2011) et à Albert Royer 50,3\% (Diouf, 2016). Cette différence pourrait s'expliquer en grande partie par la nature population d'étude. En Afrique, on note des taux de mortalité supérieurs au nôtre, mais qui restent élevés (Amri, 2008 Balaka, 2002, Cissé, 1998 Ouédraogo, 2013). Dans notre étude, l'infection était la principale circonstance de décès $(63,6 \%)$, suivie par la détresse respiratoire $(18,2 \%)$ et les hémorragies néonatales $(9,1 \%)$. En Tunisie, l'infection était responsable de $21,2 \%$ des décès ; la première cause 
de décès étant la maladie des membranes hyalines (46,2\%) (Amri, 2008). $\mathrm{Au}$ Gabon, Koko et al. avaient identifié la détresse respiratoire, au cours de l'hospitalisation, comme première cause de décès avec un taux de $60 \%$, suivie de l'infection avec $26,7 \%$ des cas (Koko, 2002). Le poids moyen à la sortie était de 1887,9 g dans notre étude, comparable au 1676g d'Ouédraogo et al. (Ouédraogo, 2013). Par contre pour Amri et al. le poids moyen à la sortie était plus élevé avec 2010g (Amri, 2008).

\section{Données sur le suivi à distance}

Nous avons retrouvé un poids médian à 1 mois de 2387,87 g inférieur à la normale. Des résultats similaires étaient retrouvés pour la taille et le périmètre crânien. A vu de ces données, on peut conclure que l'âge d'un mois soit précoce pour espérer un rattrapage. Ce rattrapage a été obtenu pour la moitié des enfants à l'âge de 30 mois pour le poids et la taille. Ce suivi à 30 mois avait permis également de noter chez un enfant qui avait présenté une asphyxie sévère des séquelles neurologiques. Ces résultats corroborent les données de la littérature selon laquelle, il existe un lien étroit entre les difficultés d'adaptation à la vie extra-utérine et le devenir neurologique (Valleur, 2004 Zupan, 2003).

\section{Conclusion}

La mortalité liée à la prématurité reste élevée dans les pays à revenu faible. La prématurité modérée occupe quasiment la moitié des cas de prématurés dans notre série. Leur prise en charge optimale passerait par une meilleure collaboration obstétrico-néonatale mais surtout par l'installation d'unités mères kangourou.

\section{References:}

1. Cissé CT, Tall-Diaw C, Sow S, Bnouhoud M, Dina G et coll. (1998). Accouchement prématuré: épidémiologie et pronostic au CHU de Dakar. J Gynecol Obstet Biol Reprod 27 : 71-76.

2. Pattinson RC. (2004). Are deaths due to prematurity avoidable in developing countries? Trop Doct. 34, 7 - 10

3. Amri F, Fatnassi R, Negra S, Khammari S et coll. (2008). Prise en charge du nouveau-né prématuré. J Pediat 21:227 - 231.

4. Bobossi-Serengbe G, Mbongo-Zunda-moyen AN, Diemer H, NadjiAdim F, Siopahtis RM et coll. (2000).

Les nouveau-nés de faible poids de naissance à l'unité de néonatologie du complexe pédiatrique de Bangui (RCA) : devenir immédiat et pronostic. Med. Afr. Noire 47(4)

5. Ballard J L. (1979). A simplified score for assessment of fetal maturation of newly born infants. J. Pediatr. 95:769 - 774. 
6. Born too soon: The Global Action Report on Preterm Birth. [En ligne]. WHO- Geneva, 2002. Consulté le 18/04/2017 Consultable à l'URL :

http://whqlibdoc.who.int/publications/2012/9789241503433_eng.pdf

7. Jeanie L C, Lex WD, et coll. (2012).

Increasing rates of prematurity and epidemiology of late preterm birth. J Paediatrics and Child Health. $48: 784$ - 788

8. Gueye M, Faye P M, Gueye Diagne N R, Seye P I, Cissé Bathily A, Bampoky P, Diallo D et coll. (2011).

Aspects épidémiologiques et évolutifs de la prématurité au Centre Hospitalier Abass Ndao de Dakar (Sénégal). Journal SAGO. 12:7-12

9. Valery S, Picone O, Cotantiee Y, Frati A, Labroussi C, Ayoubi et coll. (2015). Prématurité modérée 34 SA 637 SA description des causes et conséquences néonatales immédiates dans une maternité niveau 2. Gynécologie obstétrique et fertilité 43: $582-587$

10. Katz J, Lee Anne CC, Naoko K, Lawn J E, Simon C et coll. (2013).

Mortality risk in preterm and small-for-gestational-age infants in lowincome and middle-income countries: a pooled country analysis. Lancet: 382(9890) $417-425$.

11. Ye D, Kam Kl, Sanou I, Traoré A, Koueta F, Zeba B, Zoungran A, Sawado S et coll. (1999).

Etude épidémiologique et évolutive de la prématurité dans l'unité de néonatologie du CHN YO de Ouagadougou Ann. Pediatr. 46(9): 643 $-648$.

12. Ndiaye O, Fall AL, Dramé A, Sylla A, Gueye M, Cissé CT, Sall GM, Ba M, Kuakuvi N et coll. (2006). Facteurs étiologiques de la prématurité au centre hospitalier régional de Ziguinchor (Sénégal). Bull. Soc. Pathol. Exot. 99 (2) : $113-114$

13. Diallo F B, Diallo M S, Sylla M, Diaw S T, Diallo T S, Diallo Y, Diakité $S$ et coll. (1998) Accouchement prématuré, épidémiologie, facteurs étiologiques, stratégies préventives. Dakar méd. 43:70 - 73

14. Lejeune C. (2008). Précarité et prématurité. J Pédiatr. Puéricult. 21 : $344-348$

15. Ndiaye O, Fall A L, Gueye Ba M, Gueye A M, Diallo D, Sylla A, Gueye M, Diouf S, Sall M G, Moreau J C, Ba M, Kuakuvi N et coll.(2006). Facteurs de risqué associés au petit poids de naissance: A propos d'une étude cas-témoin à la maternité du centre hospitalier de Thiès (Sénégal).J de pédiatr. puéricult 19: 153 - 158

16. Ouedraogo/ Yugbare S O, Kabore R, Koueta F, Sawadogo H, Dao L et coll. (2013). Facteurs de risque de décès des nouveau-nés de faible poids de naissance à Ouagadougou. J. Pédiatr. Puéricult. 2013; 26 : $204-209$ 
17. Balaka B, Baeta S, Agbere A D, Boko K, Kessie K, Assimadi K et coll. (2002). Facteurs de risque associés à la prématurité au centre hospitalier universitaire de Lomé (Togo). Bull soc. Pathol. Exot 94: $208-283$.

18. Caroline HDF, Harshpal SS, Clive O, Restrepo-Mendez MC, Cesar Victora, et coll. (2015). Association between maternal age at childbirth and child and adult outcomes in the off spring: a prospective study in five low-income and middle-income countries (COHORTS collaboration). Lancet Glob Health 3: 366 - 377.

19. Prazuck T, Tall F, Roisin AJ, Konfe S, Cat M \&Lafaix C et coll.(1993). Risk factors for preterm delivery in Burkina Faso (west Africa). Int. J. Epidemiol. 22, 489 - 494.

20. Ezechi OC, Makinde ON, Kalu BE, Nnatu SN et coll.(2003). Risk factors for preterm delivery in southwestern Nigeria. J Obstet Gynaecol, 2:387 - 391

21. Diouf FN, Faye PM, Gueye M, BA ID, Guissé AL et coll. (2016). Aspects socio - démographiques épidémiologiques, cliniques et pronostiques du prématuré dans l'unité de néonatologie de l'hôpital d'enfant Albert Royer de Dakar. Rev. CAMES Sante 4 (2) ; 19 - 24

22. Enquête démographique et de santé a indicateurs multiples, Sénégal (EDS-MICS) 2010-2011 Rapport final. Agence Nationale de la Statistique et de la Démographie (ANSD) Dakar (Sénégal).MEASURE DHS, ICF International Calverton, Maryland, USA. 2012 Fév.

23. Koko J,Duffillot D, Gahouma D, Moussavou A et coll.(2002). Facteurs de mortalité des prématurés dans le service de pédiatrie de l'hôpital pédiatrique d'Owendo-Libreville (Gabon). Arch. Pédiatr. 9: 655

24. Valleur D., Magny J F., Rigourd V., Kieffer F et coll. (2004). Le pronostic neurologique à moyen et long terme des prématurés d'âge gestationnel inférieur à $28 \mathrm{SA}$ : Journal Gynécologique obstétrique et biologique de la reproduction janvier 33 : 72-78.

25. Zupan - Simunek V., Razafimahefa H., Ceymaex L et coll. (2003). Asphyxie per-partum du nouveau-né à terme pronostic neurologique des asphyxie périnatales à terme. Journal Gynécologique obstétrique et biologique de la reproduction. 32(1) : 190. 